ARTICLE

https://doi.org/10.1038/s41467-020-18274-2

\title{
Photo-mediated selective deconstructive geminal dihalogenation of trisubstituted alkenes
}

\author{
Han Wang ${ }^{1,4}$, Ren Wei Toh ${ }^{1,4}$, Xiangcheng Shi ${ }^{1}$, Tonglin Wang ${ }^{2}$, Xu Cong ${ }^{1} \&$ Jie Wu $\mathbb{C}^{1,3 凶}$
}

Selective deconstructive functionalization of alkenes, other than the well-established olefin metathesis and ozonolysis, to produce densely functionalized molecular scaffolds is highly attractive but challenging. Here we report an efficient photo-mediated deconstructive germinal dihalogenation of carbon-carbon double bonds. A wide range of geminal diiodoalkanes and bromo(iodo)alkanes ( $>40$ examples) are directly prepared from various trisubstituted alkenes, including both cyclic and acyclic olefins. This $\mathrm{C}=\mathrm{C}$ cleavage is highly chemoselective and produces geminal dihalide ketones in good yields. Mechanistic investigations suggest a formation of alkyl hypoiodites from benzyl alcohols and $\mathrm{N}$-iodoimides, which undergo light-induced homolytic cleavage to generate active oxygen radical species.

\footnotetext{
${ }^{1}$ Department of Chemistry, National University of Singapore, 3 Science Drive 3, Singapore 117543, Republic of Singapore. ${ }^{2}$ College of Chemistry and Chemical Engineering, Northwest Normal University, 730070 Lanzhou, Gansu, China. ${ }^{3}$ National University of Singapore (Suzhou) Research Institute, 377 Lin Quan Street, Suzhou Industrial Park, 215123 Suzhou, Jiangsu, China. ${ }^{4}$ These authors contributed equally: Han Wang, Ren Wei Toh. ${ }^{凶}$ email: chmjie@nus.edu.sg
} 
n organic synthesis, common functionalization usually focuses on the installation or modification of functional groups without significantly changing the backbones of molecules. In stark contrast, deconstructive functionalization is attractive as it can drastically change the scaffolds of molecules to introduce new chemical space, unmask dormant functional groups, and create functionalities tethered at a predefined distance determined by ring sizes of the reactants.

The carbon-carbon double bond is one of the most fundamental functionalities in organic molecules. Various methods have been developed to convert alkenes to important intermediates and fine chemical products, which play vital roles in the fields of material science, biochemistry, pharmaceutical science, and the chemical industry ${ }^{1-8}$. Deconstructive functionalization of alkenes has been well developed to introduce two functional groups at different sites of olefins (Fig. 1a). For instance, transition-metal-catalyzed $\mathrm{C}=\mathrm{C}$ bond cleavage processes, such as olefin metathesis, have found wide application in natural product and material synthesis ${ }^{9-12}$. Ozonolysis and other similar oxidations with various organic and inorganic oxidants were robust to introduce two carbonyl derivatives from a single $\mathrm{C}=\mathrm{C}$ bond ${ }^{13-17}$. Aside from such well-established strategies, other types of deconstructive functionalization of alkenes producing densely functionalized scaffolds remain rare and challenging ${ }^{18-27}$.

Organohalides are versatile building blocks in synthetic chemistry. They are widely utilized as precursors in transitionmetal-catalyzed cross-coupling, radical reactions, nucleophilic substitutions, and metal-halide exchanges ${ }^{28-30}$. Among them, geminal dihalides represent a unique class of compounds and have been used as carbene precursors and multi-functional synthons. However, efficient synthetic pathways to synthesize geminal dihalides are quite limited, which significantly restricts the investigation and wide application of this unique family of compounds ${ }^{31-35}$. Herein, we report a direct synthetic route to geminal dihalides by photo-mediated deconstructive fragmentation of cyclic or acyclic trisubstituted alkenes (Fig. 1b).<smiles>CCCC(=O)OCC</smiles>
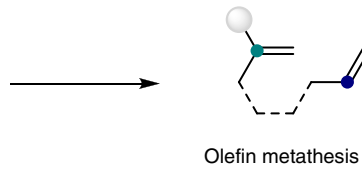

$\mathrm{Ru}, \mathrm{Mo}$, etc.

b

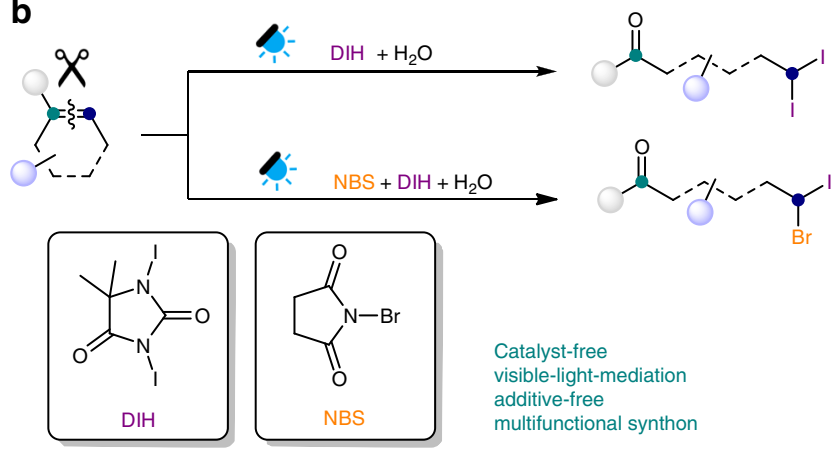

Fig. 1 Deconstructive functionalization of alkenes. a Common strategies for $\mathrm{C}=\mathrm{C}$ bond cleavage. $\mathbf{b}$ Visible-light-mediated deconstructive oxidative geminal dihalogenation of trisubstituted alkenes (this work). DIH 1,3diiodo-5,5-dimethyl-hydantoin, NBS N-bromosuccinimide, mCPBA metachloroperoxybenzoic acid.

\section{Results}

Reaction optimization. We initially designed a cascade hydrohalogenation of trisubstituted alkenes and subsequent photoinduced $\beta$-scission of the generated alcohol intermediates. Based on previous reports from groups of Chen and Zhu on photomediated conversion of alcohols to oxygen radicals ${ }^{36-42}$, our study commenced by using 1-phenyl-1-cyclohexene (1) as a model substrate. As illustrated in Table 1 , treatment of $\mathbf{1}$ with eosin Y (1 mol\%) as the photocatalyst, $N$-iodosuccinimide (NIS, 4 equiv), $\mathrm{H}_{2} \mathrm{O}$ (50 equiv) and acetoxyl benziodoxole (BIOAc, 2 equiv) in $\mathrm{MeCN}$ under blue light-emitting diode (LED) irradiation, the desired product, 6,6-diiodo-1-phenylhexan-1-one (2) was obtained in $40 \%$ yield (entry 1). Further investigation revealed that a similar result could be obtained in the absence of any photocatalyst (entry 2). To our surprise, 2 could be generated in $28 \%$ yield even without BIOAc (entry 3 ). A moderate temperature $\left(50^{\circ} \mathrm{C}\right)$ significantly accelerated the reaction (entries 4-5). Evaluation of solvents indicated that a mixed solvent of EtOAc/ $\mathrm{MeNO}_{2}(10: 1)$ was the optimal choice, leading to the generation of 2 in $81 \%$ yield (entries 6-9). Using 1,3-diiodo-5,5dimethyl-hydantoin (DIH) as the iodination agent afforded an improved yield compared to that with NIS (entry 10). Light irradiation was essential as no product $\mathbf{2}$ could be detected in the absence of light (entry 11).

Substrate scope. With the optimized conditions established, we investigated the scope of the deconstructive geminal diiodination of cyclic alkenes. As shown in Fig. 2, a variety of aryl-substituted cyclohexene derivatives underwent deconstructive geminal diiodination effectively to deliver products $\mathbf{2 - 1 5}$ in moderate to good yields. Cyclohexenes containing aryl rings with electronically distinct substituents in the ortho-, meta- or para-position afforded products 2-10 in similar yields, with the exception of a

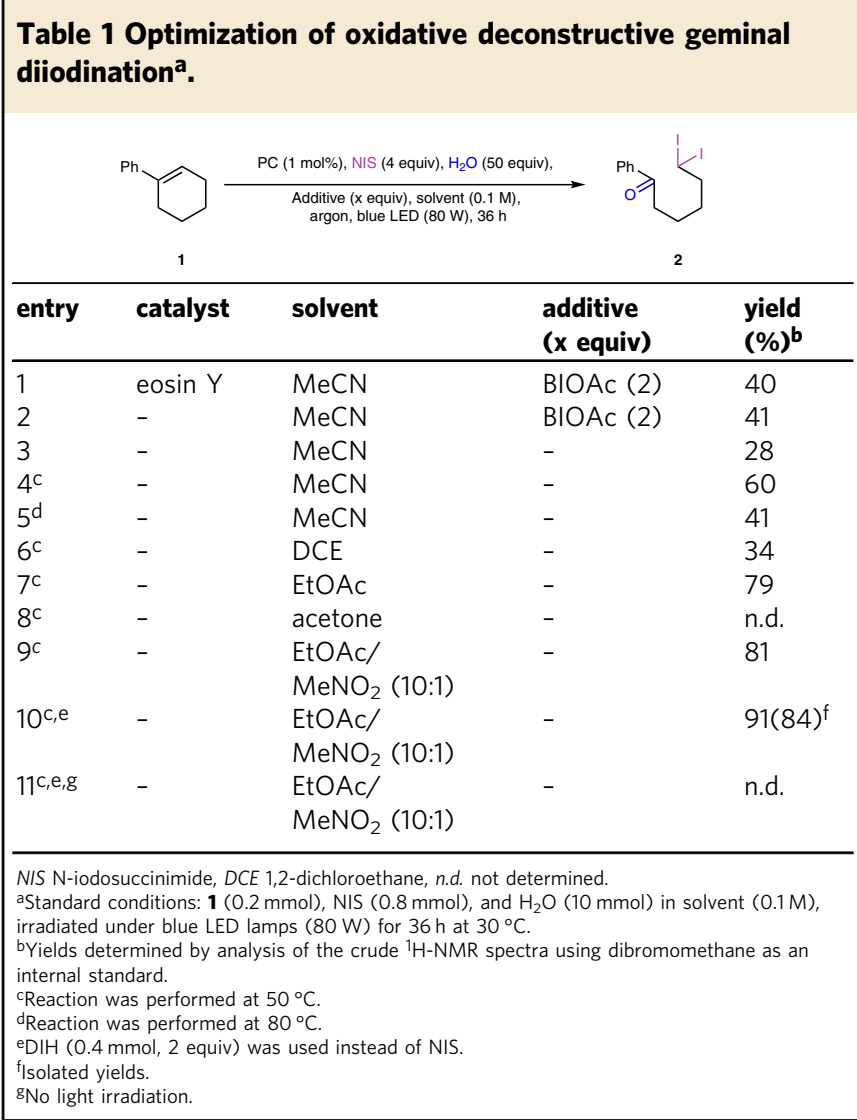




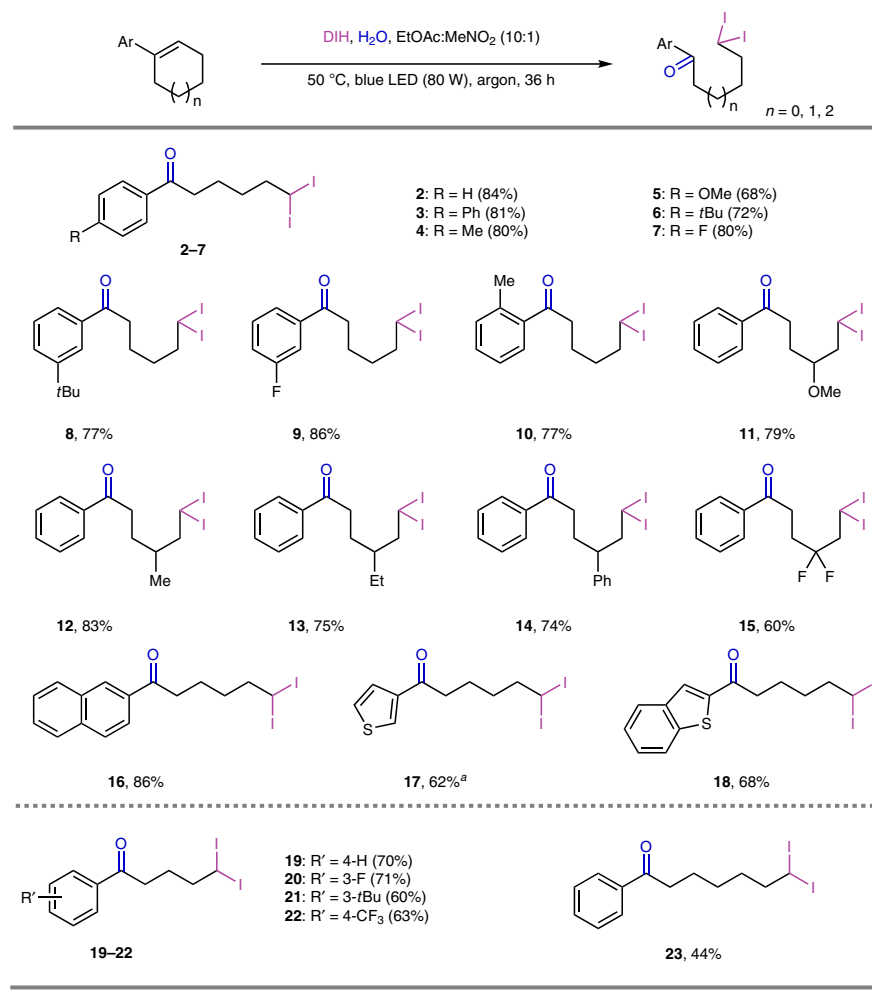

Fig. 2 Scope of oxidative deconstructive geminal diiodination of cyclic

alkenes. Isolated yields unless otherwise indicated. Performed with alkene $(0.2 \mathrm{mmol}), \mathrm{H}_{2} \mathrm{O}(10 \mathrm{mmol}), \mathrm{DIH}(0.4 \mathrm{mmol})$ in EtOAc: $\mathrm{MeNO}_{2}(10: 1)$, irradiated under blue LED lamps ( $80 \mathrm{~W}$ ) for $36 \mathrm{~h}$ at $50{ }^{\circ} \mathrm{C}$. ${ }^{a} 4$ equiv NIS instead of DIH.

compound bearing a strong electron-donating methoxy substituent, with which electrophilic aryl iodination occurred in the presence of DIH, resulting in a lower yield of product 5 . Various substituents on the cyclohexene ring, including methoxy, alkyl, phenyl, difluoro, were well tolerated, giving products 11-15 with similar yields. Naphthyl and hetero-aryl-substituted cyclohexenes were also suitable substrates, and underwent ring-opening and geminal diiodination to deliver compounds $\mathbf{1 6}-\mathbf{1 8}$ with yields of $62-86 \%$. Because the thiophene ring is very reactive towards electrophilic substitution, NIS was applied in place of DIH to avoid the iodination of the thiophene ring in the synthesis of $\mathbf{1 7}$. The deconstructive geminal diiodination is not limited to cyclohexene derivatives; a range of aryl-substituted cyclopentene and cycloheptene derivatives were all viable in this deconstructive functionalization to achieve products 19-23 in moderate to good yields. Variation of the size of the ring containing the double bond led to geminal diiodide ketone products bearing carbon chains with varying lengths.

Besides the use of DIH to generate geminal diiodo products, this strategy can be further expanded to produce potentially more useful bromo(iodo)alkanes by modifying the optimal reaction conditions by addition of NBS (Fig. 3). This cascade transformation was applied to a variety of aryl-substituted cyclic alkenes to afford the corresponding geminal bromo-iodination products. A variety of functional groups on the aryl rings or the cyclohexene scaffolds were well tolerated and delivered products 24-36 in moderate to good yields. 3,6-Dihydropyran was a good substrate for this cascade reaction thus further enriching the skeletal diversification (37). The robustness of this strategy was further explored with heteroaryl rings and cyclopentenes which generated the corresponding bromo-iodide arylketone products 38-40 in moderate yields. However, 1-phenyl-1-cycloheptene did not

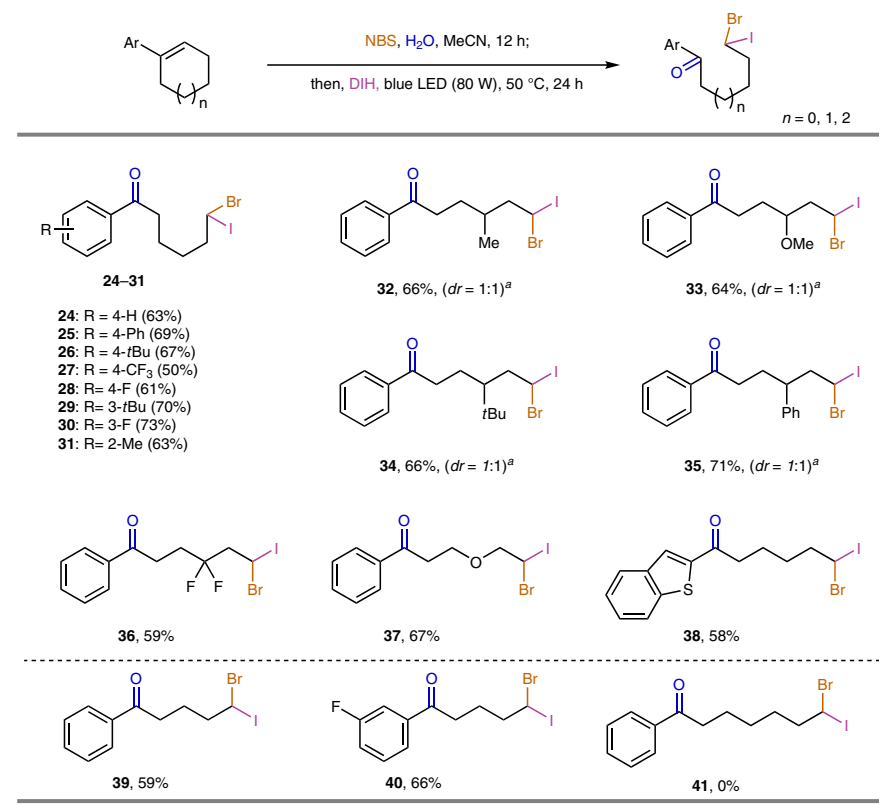

Fig. 3 Scope of oxidative deconstructive geminal bromo-iodination of cyclic alkenes. Isolated yields unless otherwise indicated. Performed with alkene $(0.2 \mathrm{mmol})$, NBS $(0.21 \mathrm{mmol}), \mathrm{H}_{2} \mathrm{O}(10 \mathrm{mmol}), \mathrm{DIH}(0.3 \mathrm{mmol})$ in $\mathrm{MeCN}$. See Supplementary Information for experimental details. ${ }^{a} \mathrm{Dr}$ values were determined by analysis of ${ }^{1} \mathrm{H}$ NMR spectra of the crude product mixture.

afford the corresponding bromo(iodo)alkane product $\mathbf{4 1}$ under our reaction conditions.

As illustrated in Fig. 4a, trialkyl-substituted alkene $\mathbf{4 2}$ was subjected to the standard conditions for geminal diiodination and bromo-iodination, but no corresponding product could be generated. A high temperature $\left(80^{\circ} \mathrm{C}\right)$ could not promote geminal diiodination of $\mathbf{4 2}$ either, while bromo-iodination product 43 could be isolated in $21 \%$ yield. However, further increasing the temperature to $100^{\circ} \mathrm{C}$ afforded only a trace amount of 43. Control experiments (Supplementary Fig. 2) indicated the instability of halohydrins at high temperatures and the ineffectiveness of trialkyl-substituted halohydrins in the deconstructive iodination step. These results highlighted the importance of a suitable temperature and aromatic substituents for the success of the deconstructive geminal dihalogenation. To further expand the scope of this reaction, acyclic trisubstituted olefins were evaluated. As shown in Fig. 4b, acyclic alkenes 44-46 with different substituent patterns were subjected to the optimal diiodination conditions, and only the gem-alkyl-aryl-substituted olefin 46 delivered the corresponding diiodide product 47 in a useful yield (47\%). Further tuning of the electronic properties in the vinyl aryl substituent (48 and 49 ) resulted in product 47 with similar yields. The deconstructive geminal diiodination proceeded readily with alkenes bearing different remote functionalities (50 and 52), affording products 51 and 53, respectively, in moderate yields.

Mechanistic elucidation with supporting evidence. Various control experiments were performed to elucidate the reaction mechanism. First, 1 was treated with DIH or NBS and water in the absence of light to afford iodohydrin $\mathbf{5 4}$ or bromohydrin $\mathbf{5 5}$, respectively. Tertiary alcohols $\mathbf{5 4}$ and $\mathbf{5 5}$ were subjected to the standard light-promoted conditions and afforded products 2 and 24, respectively, but gave no product in the absence of light (Fig. 5a). These control experiments showed that the iodohydrin and bromohydrin were intermediates in the cascade one-pot 
a
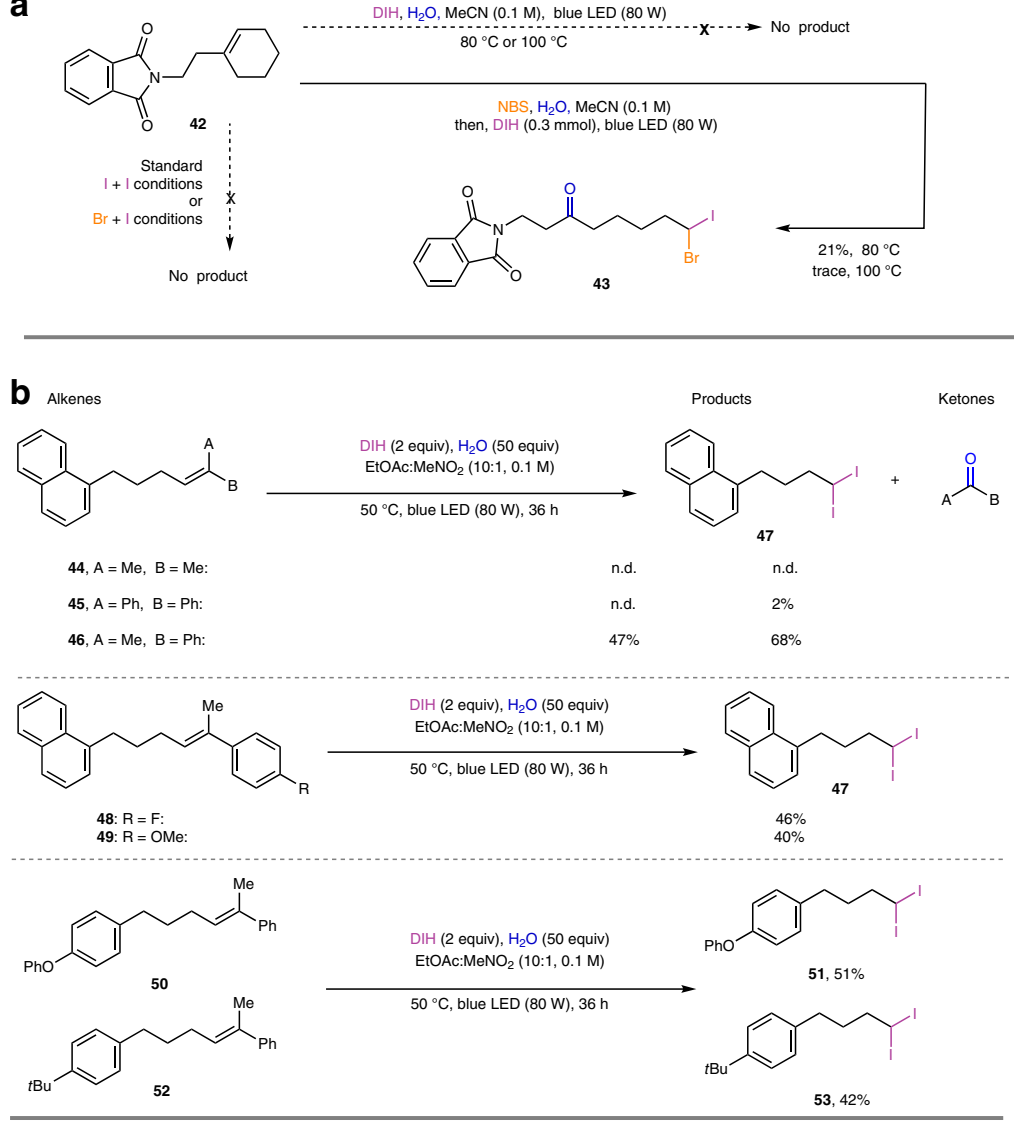

Fig. 4 Investigation of other trisubstituted alkenes. a Trialkyl-substituted cyclohexenes. b Acyclic trisubstituted alkenes.

reaction. When TEMPO was added into the reaction mixture, no dihalide product could be detected, which supported a radical based reaction mechanism. ESR measurements also indicated the presence of radical species when reacting DIH with $\mathbf{5 5}$ in MeCN under light irradiation (Supplementary Figs. 9 and 10).

Based on previous literatures ${ }^{43}$, alcohols can react with acetyl hypoiodite (AcOI) to generate alkyl hypoiodites. The O-I bonds of the alkyl hypoiodites could undergo homolytic cleavage under light irradiation to deliver a transient alkoxy radical species. We, therefore, prepared $\mathbf{5 6}$ by treating $\mathbf{5 5}$ with acetyl hypoiodite in dark, and generation of the unstable intermediate 56 was confirmed by ${ }^{1} \mathrm{H}$ NMR analysis of the reaction mixture (Supplementary Fig. 3). Irradiation of $\mathbf{5 6}$ formed in situ with blue LED lamps for $10 \mathrm{~min}$ afforded product 24 in $26 \%$ yield. When a mixture of $\mathbf{5 5}$ and $\mathrm{DIH}$ in $\mathrm{MeCN}$, reacting in the dark, was examined by ${ }^{1} \mathrm{H}$ NMR and GC-MS analysis, 56 and 1-iodo5,5-dimethylhydantoin were detected (Supplementary Fig. 4-6). This result indicates the generation of an alkyl hypoiodite from a phenyl-substituted alcohol and DIH. Even though we cannot fully exclude the possibility of formation of an electron donor-acceptor (EDA) complex between 55 and DIH, UV-Vis measurements of different stoichiometry between $\mathbf{5 5}$ and DIH did not follow the curve of Job's plot, which is normally observed for an EDA complex (Fig. 5b). Light on/off experiments indicated that a long radical chain process is unlikely. As illustrated in Fig. $5 c$, the product formation ceased immediately when the light source was periodically switched off and resumed when light was turned on.

Based on existing related literatures ${ }^{43-47}$ and all the experimental results described above, a plausible mechanism was proposed and is described in Fig. 5d. The cascade reaction is initiated by hydroxyhalogenation of alkene $\mathbf{I}$ in the presence of water and an electrophilic halogen source ${ }^{44}$. The resulting halohydrin II reacts with $\mathrm{DIH}$ to deliver the alkyl hypoiodite intermediate III. Light irradiation induces the homolysis of the labile $\mathrm{I}-\mathrm{O}$ bond to generate the reactive oxygen radical species IV $^{43,45,46}$. Carbon radical $\mathbf{V}$ is formed by $\beta$-scission of radical IV and subsequent iodination with the iodine radical or DIH accomplishes product $\mathbf{V I}^{43,46-51}$.

To further support the proposed ring-opening iodination, density functional theory (DFT) calculations were conducted on the model reaction of $\mathbf{5 5}$ with DIH. The resulting energy profiles of the reaction process are displayed in Fig. 6. The complexation of 55 and DIH leads to a zwitterionic intermediate Int1 through TS1 with active free energy of $32.6 \mathrm{kcal} / \mathrm{mol}$. This relatively high active free energy may explain why an elevated temperature is essential for an efficient transformation (Table 1, entry 3 vs 4). The iodine atom between the two carbonyl groups is more electrophilic than the other iodine atom in DIH, and undergoes complexation with alcohol 55. The subsequent heterolysis of the $\mathrm{O}-\mathrm{I}$ bond occurs together with the alcohol deprotonation through a transition state TS2 to give $\mathbf{5 6}$ and dimethyliodohydantoin with a barrier of $24.4 \mathrm{kcal} / \mathrm{mol}$ relative to Int1. Under blue light excitation, the ground-state $\mathbf{5 6}\left(\mathbf{S}_{\mathbf{0}}\right)$ is pumped to the first singlet excited-state $5 \mathbf{6}^{*}\left(\mathbf{S}_{\mathbf{1}}\right)$ with excitation energy of $66.3 \mathrm{kcal} / \mathrm{mol}$ $(2.87 \mathrm{eV})$ according to time-dependent density functional theory (TD-DFT) calculations. Homolytic cleavage of the O-I bond in 56* produces the active oxygen radical Int2. The subsequent $\beta$-scission is a facile process with a barrier of $8.5 \mathrm{kcal} / \mathrm{mol}$ to give the transient carbon radical Int3, followed by exergonic trapping with the iodine radical to accomplish the generation of product 
a

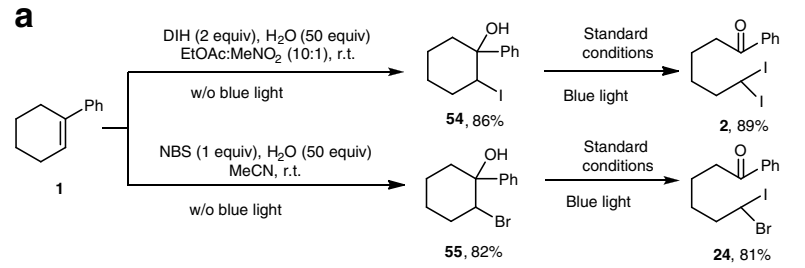

$\overbrace{54 / 55}^{\mathrm{OH}} \frac{\text { Standard conditions }}{\text { In dark }}$ No product

$\underbrace{\mathrm{Ph}}_{1}$ or $\overbrace{54 / 55}^{\mathrm{OH}} \stackrel{\begin{array}{c}\text { Standard conditions } \\ \text { blue LED }\end{array}}{\mathrm{Ph}}$ No product

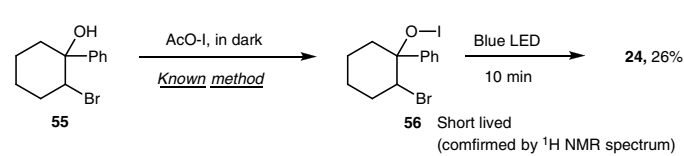

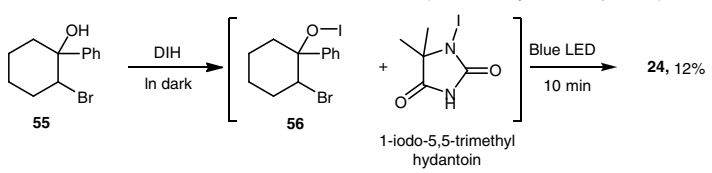

b

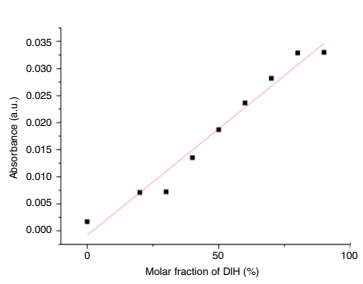

C

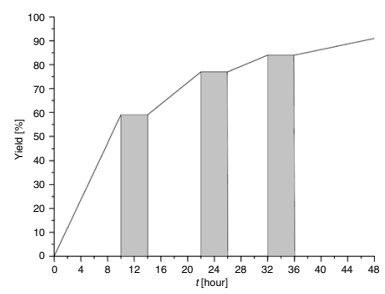

d
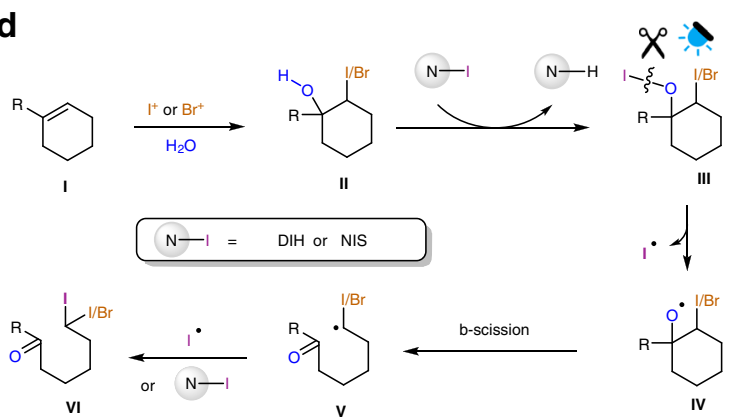

Fig. 5 Plausible mechanism with supporting evidence. a Control experiments. b UV-Vis measurements of stoichiometry between $\mathbf{5 5}$ and DIH @ $450 \mathrm{~nm}$. c Light on-off experiments of deconstructive geminal diiodination of 1. d Plausible mechanism.

a

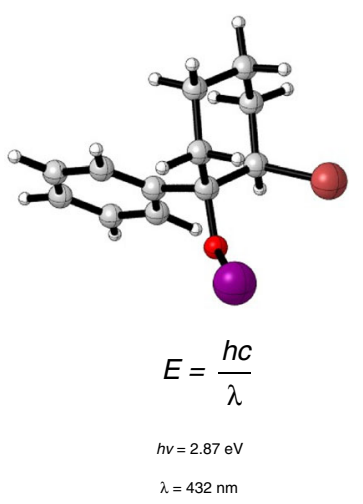

b

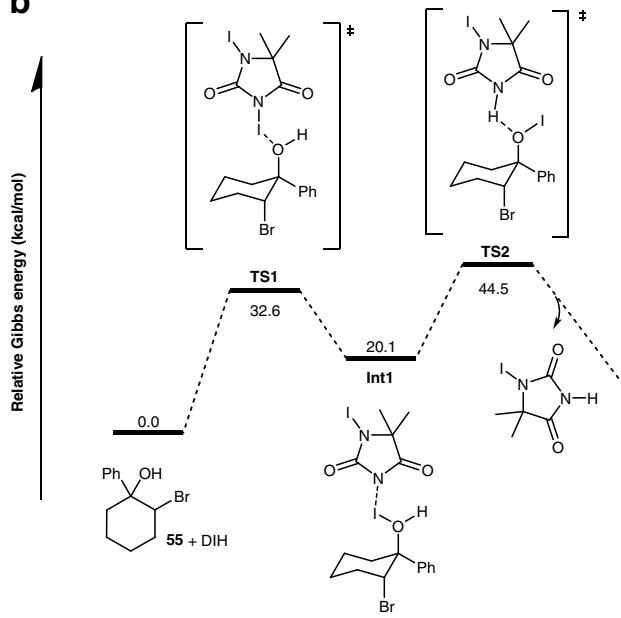

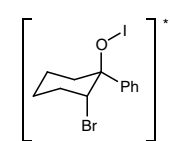
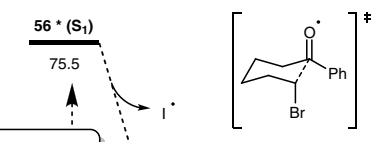

$\frac{\mathrm{TS} 3}{38.3}$

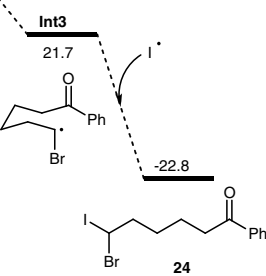

Fig. 6 DFT calculations for the ring-opening iodination of $\mathbf{5 5}$ with DIH. a Optimized geometry of $\mathbf{5 5}$ based on DFT calculation. $\mathbf{b}$ Free energy profiles for the ring-opening iodination.

24. The geometry of the unstable alkyl hypoiodite 56 was optimized and the calculated maximum light absorption is at 432 $\mathrm{nm}$ (Fig. 6a), which is close to the blue LED maximum emission $(456 \mathrm{~nm})$ used in this study.

Further synthetic applications. The oxidative geminal diiodination reaction could be achieved on gram quantities by prolonging the reaction time (Fig. 7a). To further demonstrate the synthetic utility of our methods, derivatization of generated geminal dihalide products was attempted (Fig. 7b). The diiodo compound $\mathbf{2}$ could be easily converted to disubstituted alkene $\mathbf{5 7}$ with moderate stereoselectivity by Takai-Utimoto olefination ${ }^{52,53}$. Base-promoted elimination of $\mathbf{2}$ led to vinyl iodide $\mathbf{5 8}$ in excellent yield with a moderate $E / Z$ selectivity ${ }^{54}$. Synthetically useful geminal bis(boronate) (59) was easily produced by subjecting the diiodo compound 2 to copper-catalyzed boronation ${ }^{55}$. A photoredox catalyzed deiodination using Hantzsch ester as the hydrogen source reduced $\mathbf{2}$ to $\mathbf{6 0}$ bearing an unsubstituted alkyl chain. More interestingly, highly selective derivatization of the iodide in the bromo(iodo)alkane products could be achieved. The photoredox reaction using Hantzsch ester converted 24 to alkylbromide $\mathbf{6 1}$ in $90 \%$ yield. Based-promoted elimination of $\mathbf{2 4}$ selectively produced the corresponding vinyl bromide 62. Bromoiodide 24 could undergo a selective $\mathrm{S}_{\mathrm{N}} 2$ reaction with sodium azide to afford 63 in $86 \%$ yield. Subjection of this azide (63) to copper-catalyzed azide-alkyne cycloaddition afforded a bromo triazole ${ }^{56}$, which could undergo further nucleophilic substitution to deliver $\alpha$-functionalized triazole $\mathbf{6 4}$ in $69 \%$ yield.

\section{Discussion}

In summary, we herein report a simple protocol for deconstructive geminal diiodination and bromo-iodination of trisubstituted alkenes under visible light irradiation. The success of this transformation relies on the formation of a labile alkyl hypoiodite 

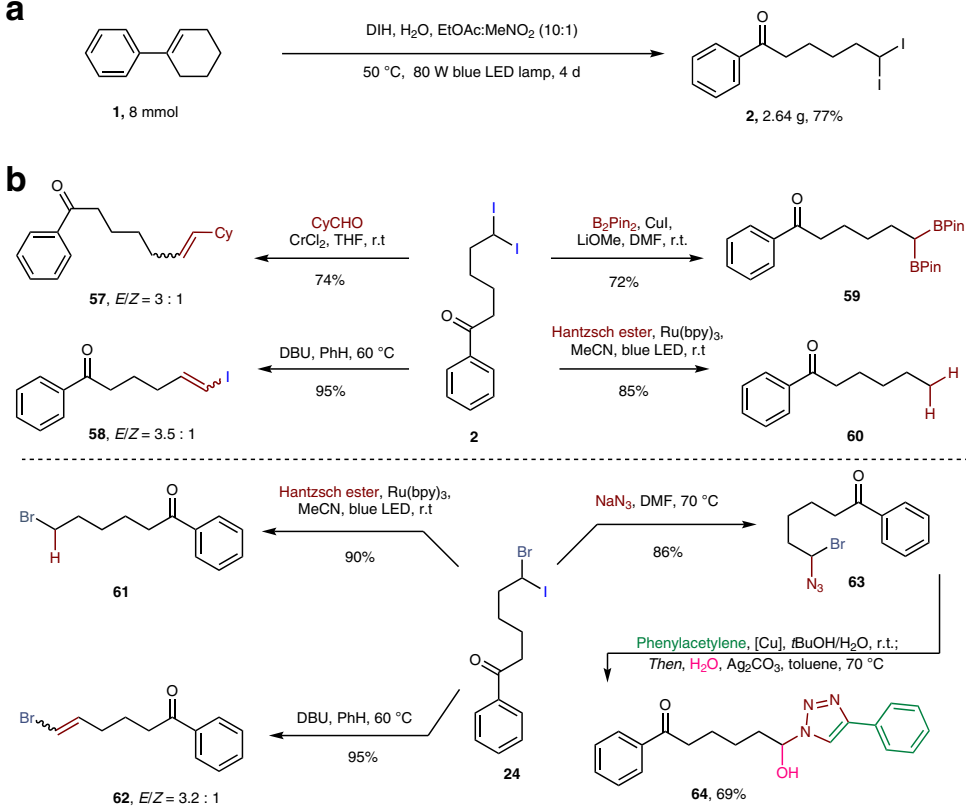

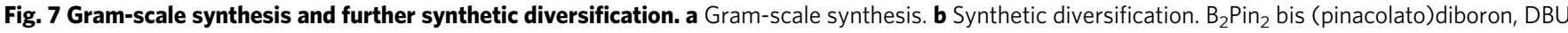
1,8-diazabicyclo (5.4.0)undec- 7-ene.

intermediate between the halohydrin and DIH. The protocol is distinguished by its operational simplicity, metal-free and catalyst-free characters, a wide scope of both cyclic and acyclic alkenes, delivery of useful and otherwise difficultly accessible synthons, and controllable chain length of products by choosing alkenes with different ring sizes.

\section{Methods}

General procedure of the deconstructive geminal diiodination. Alkene $(0.2$ mmol, 1 equiv) and 1,3-diiodo-5,5-dimethylhydantoin ( $0.4 \mathrm{mmol}, 2$ equiv), $\mathrm{H}_{2} \mathrm{O}$ (10 mmol, 50 equiv), and EtOAc: $\mathrm{MeNO}_{2}(10: 1,2 \mathrm{~mL})$ were added to a schlenk tube $(10 \mathrm{~mL})$ equipped with a magnetic stirring bar. Then, the reaction mixture was operated by freeze-pump-thaw procedures three times and backfilled with argon. The resulting solution was irradiated by blue LED lamps $(2 \times 40 \mathrm{~W})$ and magnetically stirred at $50^{\circ} \mathrm{C}$. After $36 \mathrm{~h}$, the reaction solution was concentrated, and the product was purified by column chromatography $\left(\mathrm{SiO}_{2}\right)$. The diastereomeric ratio was determined by ${ }^{1} \mathrm{H}$ NMR of the crude product mixture. See Supplementary Methods for details.

General procedure of the deconstructive bromo-iodination. Alkene $(0.2 \mathrm{mmol})$ and $N$-bromosuccinimide ( $0.21 \mathrm{mmol}, 1$ equiv), $\mathrm{H}_{2} \mathrm{O}$ (10 mmol, 50 equiv), and $\mathrm{MeCN}(2 \mathrm{~mL})$ were added to a schlenk tube $(10 \mathrm{~mL})$ equipped with a magnetic stirring bar. The reaction mixture was operated by freeze-pump-thaw procedures for three times and backfilled with argon. The resulting solution was magnetically stirred at $50^{\circ} \mathrm{C}$ for $12 \mathrm{~h}$. Then, 1,3-diiodo-5,5-dimethylhydantoin $(0.3$ mmol, 1.5 equiv) was added to the reaction mixture under argon. The reaction mixture was irradiated by blue LED lamps $(2 \times 40 \mathrm{~W})$ and magnetically stirred at $50{ }^{\circ} \mathrm{C}$. After $24 \mathrm{~h}$, the reaction solution was concentrated, and the product was purified by column chromatography $\left(\mathrm{SiO}_{2}\right)$. The diastereomeric ratio was determined by ${ }^{1} \mathrm{H}$ NMR of the crude product mixture. See Supplementary Methods for details.

Computational details. Density functional theory (DFT) calculations were performed for the verification of the mechanism. The geometries optimization in this study (except 56 and 56*) was performed at the (u)B3LYP-D3(BJ) level of theory. The $6-311+g(d, p)$ basis set was used for all $\mathrm{H}, \mathrm{C}, \mathrm{N}$, and $\mathrm{O}$ atoms, and the Stuttgart-Dresden basis set (SDD) was employed for $\mathrm{Br}$ and I atoms. The nature of the stationary points (minima with no imaginary frequency or transition states with one imaginary frequency) was confirmed. The free energies of the optimized geometries were calculated at the same level of theory, taking into account the solvent effect of acetonitrile using Solvent Polarizable Continuum Model (PCM). Unless specified otherwise, the Gibbs free energy was used throughout. Considering the deviation in the free energies is $\sim 1.89 \mathrm{kcal} / \mathrm{mol}$ from the standard state ( $1 \mathrm{~atm}$ ) to $1 \mathrm{M}$ in solution, we reduced by $1.89 \mathrm{kcal} / \mathrm{mol}$ to the free energy for additional steps and added by $1.89 \mathrm{kcal} / \mathrm{mol}$ for the dissociation steps ${ }^{57}$. For transition state, intrinsic reaction coordinate (IRC) calculations were performed to verify whether it connected with correct reactants and products or intermediates. Time-dependent density functional theory (TD-DFT) was performed to calculate the vertical excitation energies of the photodissociation process, using the CAM-B3LYP-D3(BJ) ${ }^{58}$ level of theory with the same basis set. All calculations were performed using the Gaussian 16 Rev. A.03 software suite ${ }^{59}$. The geometries were realized using CYLview, $1.0^{60}$.

\section{Data availability}

The authors declare that all other data supporting the findings of this study are available within the article and Supplementary Information files, and also are available from the corresponding author upon reasonable request.

Received: 13 May 2020; Accepted: 12 August 2020; Published online: 08 September 2020

\section{References}

1. Hoveyda, A. H., Evans, D. A. \& Fu, G. C. Substrate-directable chemical reactions. Chem. Rev. 93, 1307-1370 (1993).

2. Grubbs, R. H. \& Chang, S. Recent advances in olefin metathesis and its application in organic synthesis. Tetrahedron 54, 4413-4450 (1998).

3. McDonald, R. I., Liu, G. \& Stahl, S. S. Palladium(II)-catalyzed alkene functionalization via nucleopalladation: stereochemical pathways and enantioselective catalytic applications. Chem. Rev. 111, 2981-3019 (2011).

4. Vasseur, A., Bruffaerts, J. \& Marek, I. Remote functionalization through alkene isomerization. Nat. Chem. 8, 209-219 (2016).

5. Dong, Z., Ren, Z., Thompson, S. J., Xu, Y. \& Dong, G. Transition-metalcatalyzed C-H alkylation using alkenes. Chem. Rev. 117, 9333-9403 (2017).

6. Lan, X.-W., Wang, N.-X. \& Xing, Y. Recent advances in radical difunctionalization of simple alkenes. Eur. J. Org. Chem. 39, 5821-5851 (2017).

7. Lee, J. H., Choi, S. \& Hong, K. B. Alkene difunctionalization using hypervalent iodine reagents: progress and developments in the past ten years. Molecules 24, 2634-2657 (2019)

8. Lin, J., Song, R.-J., Hu, M. \& Li, J.-H. Recent advances in the intermolecular oxidative difunctionalization of alkenes. Chem. Rec. 19, 440-451 (2019).

9. Grubbs, R. H., Miller, S. J. \& Fu, G. C. Ring-closing metathesis and related processes in organic synthesis. Acc. Chem. Res. 28, 446-452 (1995).

10. Hoveyda, A. H. \& Zhugralin, A. R. The remarkable metal-catalysed olefin metathesis reaction. Nature 450, 243-251 (2007).

11. Grubbs, R. H. \& O'Leary, D. J. Handbook of metathesis, Volume 2: Applications in Organic Synthesis, 2nd edn. (Wiley-VCH, Weinhein, 2015).

12. Shen, X. et al. Kinetically $E$-selective macrocyclic ring-closing metathesis Nature 541, 380-385 (2017) 
13. Bailey, P. S. The reactions of ozone with organic compounds. Chem. Rev. 58, 925-1010 (1958).

14. Ornum, S. G. V., Champeau, R. M. \& Pariza, R. Ozonolysis applications in drug synthesis. Chem. Rev. 106, 2990-3001 (2006).

15. Daw, P. et al. A highly efficient catalyst for selective oxidative scission of olefins to aldehydes: abnormal-NHC-Ru(II) complex in oxidation chemistry. J. Am. Chem. Soc. 136, 13987-13990 (2014).

16. Wang, T., Jing, X., Chen, C. \& Yu, L. Organoselenium-catalyzed oxidative $\mathrm{C}=\mathrm{C}$ bond cleavage: a relatively green oxidation of alkenes into carbonyl compounds with hydrogen peroxide. J. Org. Chem. 82, 9342-9349 (2017)

17. Yu, W. \& Zhao, Z. Catalyst-free selective oxidation of diverse olefins to carbonyls in high yield enabled by light under mild conditions. Org. Lett. 21, 7726-7730 (2019).

18. Wang, T. \& Jiao, N. TEMPO-catalyzed aerobic oxygenation and nitrogenation of olefins via $\mathrm{C}=\mathrm{C}$ double-bond cleavage. J. Am. Chem. Soc. 135, 11692-11695 (2013).

19. Liu, H., Feng, M. \& Jiang, X. Unstrained carbon-carbon bond cleavage. Chem. Asian J. 9, 3360-3389 (2014).

20. Zong, X., Zheng, Q.-Z. \& Jiao, N. NBS mediated nitriles synthesis through $\mathrm{C}=\mathrm{C}$ double bond cleavage. Org. Biomol. Chem. 12, 1198-1202 (2014).

21. Chen, W.-L. et al. Synthesis of 2-aminobenzonitriles through nitrosation reaction and sequential iron(III)-catalyzed C-C bond cleavage of 2 arylindoles. Org. Lett. 20, 3527-3530 (2018).

22. Sivaguru, P., Wang, Z., Zanoni, G. \& Bi, X. Cleavage of carbon-carbon bonds by radical reactions. Chem. Soc. Rev. 48, 2615-2656 (2019).

23. Li, J., Wei, J., Zhu, B., Wang, T. \& Jiao, N. Cu-catalyzed oxygenation of alkenetethered amides with $\mathrm{O}_{2}$ via unactivated $\mathrm{C}=\mathrm{C}$ bond cleavage: a direct approach to cyclic imides. Chem. Sci. 10, 9099-9103 (2019).

24. $\mathrm{Wu}, \mathrm{X} . \& \mathrm{Zhu}, \mathrm{C}$. Recent advances in ring-opening functionalization of cycloalkanols by C-C $\sigma$-Bond cleavage. Chem. Rec. 18, 587-598 (2018).

25. $\mathrm{Wu}, \mathrm{X}$. \& Zhu, C. Recent advances in alkoxy radical-promoted C-C and C-H bond functionalization starting from free alcohols. Chem. Commun. 55, 9747-9756 (2019).

26. Lin, R., Chen, F. \& Jiao, N. Metal-free, NHPI catalyzed oxidative cleavage of C-C double bond using molecular oxygen as oxidant. Org. Lett. 14, 4158-4161 (2012).

27. $\mathrm{Hu}, \mathrm{W}$. et al. Palladium-catalyzed intermolecular oxidative coupling reactions of $(Z)$-enamines with isocyanides through selective $\beta-\mathrm{C}\left(\mathrm{sp}^{2}\right)-\mathrm{H}$ and/or $\mathrm{C}=\mathrm{C}$ bond cleavage. Chin. J. Chem. 36, 712-715 (2018).

28. Hughes, E. D. Reactions of halides in solution. Q. Rev. Chem. Soc. 5, 245-269 (1951).

29. Saikia, I., Borah, A. J. \& Phukan, P. Use of bromine and bromo-organic compounds in organic synthesis. Chem. Rev. 116, 6837-7042 (2016)

30. Biffis, A., Centomo, P., Zotto, A. D. \& Zecca, M. Pd metal catalysts for crosscouplings and related reactions in the 21 st century: a critical review. Chem. Rev. 118, 2249-2295 (2018).

31. Conly, J. C. Applications of the hunsdiecker silver salt degradation. The preparation of dibromides and tribromides. J. Am. Chem. Soc. 75, 1148-1150 (1953).

32. Doyle, M. P. \& Siegfried, B. Oxidative deamination of primary amines: selective synthesis of geminal dihalides. J. Chem. Soc. Chem. Commun. 12, 433-434 (1976).

33. Lansinger, J. M. \& Ronald, R. C. Reactions of aromatic aldehydes with boron halides. Synth. Commun. 9, 341-349 (1979).

34. Huan, Z., Landgrebe, J. A. \& Peterson, K. Deoxygenation of aldehydes and ketones, a new general reaction of dibromocarbene and dibromocarbonyl ylides. Tetrahedron Lett. 24, 2829-2832 (1983).

35. Cloarec, J.-M. \& Charette, A. B. Highly efficient two-step synthesis of C-sp ${ }^{3}$ centered geminal diiodides. Org. Lett. 6, 4731-4734 (2004).

36. Zhao, H., Fan, X., Yu, J. \& Zhu, C. Silver-catalyzed ring-opening strategy for the synthesis of $\beta$ - and $\gamma$-fluorinated ketone. J. Am. Chem. Soc. 137, 3490-3493 (2015).

37. Ren, R., Zhao, H., Huan, L. \& Zhu, C. Manganese-catalyzed oxidative azidation of cyclobutanols: regiospecific synthesis of alkyl azides by $\mathrm{C}-\mathrm{C}$ bond cleavage. Angew. Chem. Int. Ed. 54, 12692-12696 (2015).

38. Jia, K., Zhang, F., Huang, H. \& Chen, Y. Visible-light-induced alkoxyl radical generation enables selective $\mathrm{C}\left(\mathrm{sp}^{3}\right)-\mathrm{C}\left(\mathrm{sp}^{3}\right)$ bond cleavage and functionalizations. J. Am. Chem. Soc. 138, 1514-1517 (2016).

39. Jia, K., Pan, Y. \& Chen, Y. Selective carbonyl-C(sp3) bond cleavage to construct ynamides, ynoates, and ynones by photoredox catalysis. Angew. Chem. Int. Ed. 56, 2478-2481 (2017)

40. Wang, D., Mao, J. \& Zhu, C. Visible light-promoted ring-opening functionalization of unstrained cycloalkanols via inert C-C bond scission. Chem. Sci. 9, 5805-5809 (2018).

41. $\mathrm{Wu}, \mathrm{X}$. et al. Metal-free alcohol-directed regioselective heteroarylation of remote unactivated C(sp3)-H bonds. Nat. Commun. 9, 3343-3351 (2018).
42. Ji, M., Wu, Z. \& Zhu, C. Visible-light-induced consecutive C-C bond fragmentation and formation for the synthesis of elusive unsymmetric 1,8 dicarbonyl compounds. Chem. Commun. 55, 2368-2371 (2019).

43. Beebe, T. et al. Oxidation of alcohols with acetyl hypoiodite. J. Org. Chem. 40, 1992-1994 (1975)

44. Yang, Y., Su, C., Huang, X. \& Liu, Q. Halohydroxylation of alkylidenecyclopropanes using $N$-halosuccinimide (NXS) as the halogen source: an efficient synthesis of halocyclopropylmethanol and 3-halobut-3-en1-ol derivatives. Tetrahedron Lett. 50, 5754-5756 (2009)

45. Janjatovic, J. \& Majerski, Z. Synthesis of adamantanoid ketones from bridgehead alcohols by the hypoiodite thermolysis-cyclization sequence. $J$. Org. Chem. 45, 4892-4898 (1980)

46. Courtneidge, J. L., Lusztyk, J. \& Pagé, D. Alkoxyl radicals from alcohols. Spectroscopic detection of intermediate alkyl and acyl hypoiodites in the Suárez and Beebe reactions. Tetrahedron Lett. 35, 1003-1006 (1994).

47. Suginome, H., Takeda, T., Itoh, M., Nakayama, Y. \& Kobayashi, K. Photoinduced molecular transformations. Part 152. Ring expansion based on a sensitized $[2+2]$ photoaddition of enol ethers of cyclic ketones with olefins, followed by a $\beta$-scission of alkoxyl radicals generated from the resulting cyclobutanols. Two-carbon ring expansion of $\beta$-indanone, $\beta$-tetralone and $\beta$ suberone. J. Chem. Soc. Perkin Trans. 1, 49-61 (1995).

48. Guo, J.-J. et al. Photocatalytic $\mathrm{C}-\mathrm{C}$ bond cleavage and amination of cycloalkanols by cerium(III) chloride complex. Angew. Chem. Int. Ed. 55, 15319-15322 (2016)

49. Shi, J.-L., Wang, Y., Wang, Z., Dou, B. \& Wang, J. Ring-opening iodination and bromination of unstrained cycloalkanols through $\beta$-scission of alkoxy radicals. Chem. Commun. 56, 5002-5005 (2020)

50. Roque, J. B., Kuroda, Y., Göttemann, L. T. \& Sarpong, R. Deconstructive diversification of cyclic amines. Nature 564, 244-248 (2018).

51. Wang, Y. et al. Visible-light-promoted site-specific and diverse functionalization of a $\mathrm{C}\left(\mathrm{sp}^{3}\right)-\mathrm{C}\left(\mathrm{sp}^{3}\right)$ bond adjacent to an arene. ACS Catal. 10, 6603-6612 (2020)

52. Takai, K., Nitta, K. \& Utimoto, K. Simple and selective method for aldehydes $(\mathrm{RCHO}) \rightarrow(E)$-haloalkenes ( $\mathrm{RCH}: \mathrm{CHX}$ ) conversion by means of a haloformchromous chloride system. J. Am. Chem. Soc. 108, 7408-7410 (1986).

53. Okazoe, T., Takai, K. \& Utimoto, K. (E)-Selective olefination of aldehydes by means of gem-dichromium reagents derived by reduction of gemdiiodoalkanes with chromium(II) chloride. J. Am. Chem. Soc. 109, 951-953 (1987).

54. Martínez, A. G., Alvareza, R. M., González, S. M., Subramanian, L. R. \& Conrad, M. A new procedure for the synthesis of (E)-1-iodo-1-alkenes. Tetrahedron Lett. 33, 2043-2044 (1992).

55. Zhang, Z.-Q. et al. Copper-catalyzed/promoted cross-coupling of gemdiborylalkanes with nonactivated primary alkyl halides: an alternative route to alkylboronic esters. Org. Lett. 16, 6342-6345 (2014).

56. Himo, F. et al. Copper(I)-catalyzed synthesis of azoles. DFT study predicts unprecedented reactivity and intermediates. J. Am. Chem. Soc. 127, 210-216 (2005).

57. Zhu, C. et al. A multicomponent synthesis of stereodefined olefins via nicke catalysis and single electron/triplet energy transfer. Nat. Catal. 2, 678-687 (2019).

58. Yanai, T., Tew, D. P. \& Handy, N. C. A new hybrid exchange-correlation functional using the coulomb-attenuating method (CAM-B3LYP). Chem. Phys. Lett. 393, 51-57 (2004).

59. Frisch, M. J. et al. Gaussian 16, revision C.01 (Gaussian, 2016).

60. Legault, C. Y. CYLview 1.0 b (Unversité de Sherbrooke, 2009) http://www. cylview.org.

\section{Acknowledgements}

J.W. is grateful for the financial support provided by the Ministry of Education (MOE) of Singapore (MOE2017-T2-2-081), National University of Singapore (R-143-000-B60114), NUS (Suzhou) Research Institute and National Natural Science Foundation of China (Grant No. 21702142, 21871205). We also express our gratitude to Prof. Zhi-Jian Zhao (Tianjin University) for helpful discussions regarding the DFT calculations and Dr. Tengfei Ji (RWTH Aachen University) for assistance on mechanism study.

\section{Author contributions}

H.W. discovered and developed the reaction. H.W. and J.W. conceived and designed the investigations. X.S. conducted density functional theory (DFT) calculations. H.W., R.W.T. T.W., and X.C. performed the experiments. H.W., R.W.T. and J.W. wrote the paper.

\section{Competing interests}

The authors declare no competing interests. 


\section{Additional information}

Supplementary information is available for this paper at https://doi.org/10.1038/s41467020-18274-2.

Correspondence and requests for materials should be addressed to J.W.

Peer review information Nature Communications thanks Ning Jiao and the other, anonymous, reviewer(s) for their contribution to the peer review of this work. Peer reviewer reports are available.

Reprints and permission information is available at http://www.nature.com/reprints

Publisher's note Springer Nature remains neutral with regard to jurisdictional claims in published maps and institutional affiliations. (c) (i) Open Access This article is licensed under a Creative Commons Attribution 4.0 International License, which permits use, sharing, adaptation, distribution and reproduction in any medium or format, as long as you give appropriate credit to the original author(s) and the source, provide a link to the Creative Commons license, and indicate if changes were made. The images or other third party material in this article are included in the article's Creative Commons license, unless indicated otherwise in a credit line to the material. If material is not included in the article's Creative Commons license and your intended use is not permitted by statutory regulation or exceeds the permitted use, you will need to obtain permission directly from the copyright holder. To view a copy of this license, visit http://creativecommons.org/ licenses/by/4.0/.

(C) The Author(s) 2020 